\title{
Network Pharmacology-Based Investigation of the Therapeutic Mechanisms of Action of Danning Tablets in Nonalcoholic Fatty Liver Disease
}

\author{
Tong Lin $\mathbb{D},{ }^{1}$ Li Li $\mathbb{D},{ }^{1}$ Caijun Liang $\mathbb{D},{ }^{1}$ and Lisheng Peng $\mathbb{D}^{2}$ \\ ${ }^{1}$ The Fourth Clinical Medical School, Guangzhou University of Chinese Medicine, Shenzhen 518033, China \\ ${ }^{2}$ Shenzhen Hospital of Traditional Chinese Medicine, Shenzhen 518033, China \\ Correspondence should be addressed to Lisheng Peng; lisheng_peng@outlook.com
}

Received 8 April 2020; Revised 8 April 2021; Accepted 13 April 2021; Published 27 April 2021

Academic Editor: Weicheng $\mathrm{Hu}$

Copyright (c) 2021 Tong Lin et al. This is an open access article distributed under the Creative Commons Attribution License, which permits unrestricted use, distribution, and reproduction in any medium, provided the original work is properly cited.

Nonalcoholic fatty liver disease (NAFLD) is a rising global public health concern due to its prevalence. Danning Tablets (DNt), a composite prescription of Chinese herbal medicine, shows significant curative effects on NAFLD in clinical application. This study aimed to decipher the bioactive substances and potential mechanisms of action of DNt in the treatment of NAFLD, applying an integrated network pharmacology approach. First, the bioactive compounds of DNt were screened based on their pharmacokinetic properties, and the corresponding drug targets were predicted. Then, the NAFLD-related targets were collected. The overlapping targets between the putative targets of DNt and NAFLD-related targets were identified as the potential therapeutic targets of DNt against NAFLD. Subsequently, the networks were constructed and analyzed, and the key bioactive compounds and targets were screened out depending on their importance in the networks. Functional enrichment analysis was carried out to elucidate the potential mechanisms of DNt acting on NAFLD. Finally, a molecular docking simulation was implemented to assess the potential binding affinity between the key targets and the bioactive compounds. As a result, 43 bioactive compounds of DNt and 69 putative targets were identified. Based on the network analysis, we found seven key bioactive compounds (quercetin, $\beta$ sitosterol, luteolin, kaempferol, supraene, curcumenolactone C, and stigmasterol) of DNt might treat NAFLD via intervening IL6, MAPK8, VEGFA, CASP3, ALB, APP, MYC, PPARG, and RELA. The functional enrichment analysis revealed that DNt might affect NAFLD by modulating the signaling pathways involved in lipid metabolism, inflammation, oxidation, insulin resistance (IR), atherosclerosis, and apoptosis. Furthermore, most key bioactive compounds might bind firmly with the key targets. This study predicted the multicomponent, multitarget, and multipathway mechanisms of DNt in the treatment of NAFLD from a holistic perspective. DNt could be a promising agent for NAFLD, but further experimental verifications are still needed.

\section{Introduction}

Nonalcoholic fatty liver disease (NAFLD) is presently the most common liver disease, affecting a quarter of the adult population worldwide [1]. NAFLD, hallmarked by lipid accumulation in hepatocytes without significant alcohol intake, encompasses simple hepatic steatosis and nonalcoholic steatohepatitis (NASH), along with cirrhosis and even liver cancer developed by NASH [1]. NASH takes up 25 percent of NAFLD, and more than $1 / 3$ of NASH would die from end-stage liver disease, making it the main reason for liver transplantation $[2,3]$. Even though the pathogenesis of
NAFLD has not been fully elucidated, insulin resistance (IR), inflammation, and oxidative stress are acknowledged as the essential contributors to the onset and progression of the disease $[4,5]$. Currently, insulin sensitizers, antioxidants, lipid-lowering drugs, and lifestyle modifications are the mainly recommended treatment for NAFLD. However, there is no officially approved medication for NAFLD at present, and less than half of the patients can achieve the goal of weight loss [6,7]. As an important part of complementary and alternative treatments, herbal medicine is a promising candidate for the treatment strategies of chronic liver diseases [8]. 
Danning Tablet (DNt) is a composite Chinese patent medicine, which consists of seven Chinese herbs: Rhei Radix et Rhizome (Dahuang, RRR), Polygoni Cuspidati Rhizoma et Radix (Huzhang, PCRR), Citri Reticulatae Pericarpium Viride (Qingpi, CRPV), Imperatae Rhizoma (Baimaogen, IR), Citrus Reticulatae Pericarpium (Chenpi, CRP), Curcumae Radix (Yujin, CR), and Crataegi Fructus (Shanzha, $\mathrm{CF}$ ). DNt has been widely used to treat hepatobiliary diseases, such as cholelithiasis, chronic cholecystitis, and NAFLD for more than ten years in China, and it had obtained a product license by Canadian Natural Medicines and Over-The-Counter Medicines Agency in 2017. A multicenter clinical trial showed that DNt could improve clinical symptoms, serum ALT (alanine aminotransferase) level, and blood lipids of NAFLD patients without significant adverse events [9]. Another two clinical studies reported that DNt could reduce tumor necrosis factor $\alpha$ (TNF- $\alpha$ ) and malondialdehyde, increase superoxide dismutase in serum, and attenuate IR significantly $[10,11]$. The experimental studies demonstrated that DNt might alleviate hyperlipidemia, liver function damage, and fatty degeneration and punctiform necrosis of liver tissue in animals with NAFLD $[12,13]$. It was also reported that the lipid modulation effects of DNt might be associated with the induction of peroxisomal proliferator-activated receptor $\alpha(\operatorname{PPAR} \alpha)$ and cholesterol 7-hydroxylase (CYP7A1) in rat liver cells [13]. Besides, DNt could exert hepatoprotective effects by regulating oxidative stress, hepatic transporters, hepatic metabolic enzymes, and inflammatory signal pathways [14-16]. However, the essential compounds and the underlying mechanisms of DNt in the treatment of NAFLD have not been elucidated.

Chinese herbal medicine serves as a multicomponent, multitarget, and multipathway therapy, which makes it unique in treating complex diseases, but causes difficulty in mechanism elucidation meanwhile. As an approach integrated systems biology, bioinformatics, and polypharmacology, network pharmacology is a powerful tool to investigate complex diseases and uncover the complex interactions among drugs, targets, and diseases [13-17]. Molecular docking is a widely used in silico structure-based method to predict the interactions between molecules and biological targets, which enables the virtual screening of amounts of compounds in a limited time [18].

In this study, a network pharmacology approach was applied to explore the potential mechanisms of DNt against NAFLD. First of all, the bioactive compounds of DNt were screened according to their pharmacokinetic properties, and the corresponding targets of the bioactive compounds were predicted. Then, NAFLD-related targets were collected, the overlapping targets of DNt and NAFLD were regarded as the potential therapeutic targets of DNt against NAFLD. Furthermore, two interactive networks were built to screen out the key bioactive compounds and the key therapeutic targets. After that, functional enrichment analysis was conducted to expound the anti-NAFLD mechanisms of DNt. At last, the binding potentiality between the key target proteins and the bioactive compounds was evaluated through molecular docking. A flowchart of this study is described in Figure 1.

\section{Materials and Methods}

2.1. Screening of the Bioactive Compounds of DNt. The information of the compounds of the seven herbs in DNt was collected from Traditional Chinese Medicine Systems Pharmacology Database [19] (TCMSP, http://tcmspw.com/ index.php), Bioinformatics Analysis Tool for Molecular Mechanisms of Traditional Chinese Medicine Database (BATMAN-TCM, http://bionet.ncpsb.org/batman-tcm/), Natural Product Activity \& Species Source Database (NPASS, http://bidd2.nus.edu.sg/NPASS/), and Traditional Chinese Medicine Information Database [20] (TCM-ID, http://bidd.nus.edu.sg/group/TCMsite/Default.aspx).

Oral bioavailability $(\mathrm{OB})$ is the percentage of an orally administered dose of medicine that reaches the circulatory system; compounds with a higher $\mathrm{OB}$ value signify a better capability of being oral drugs [21]. Intestinal epithelial permeability (IEP) measures the intestinal absorption of drugs, which is partly responsible for the bioavailability of oral drugs [22]. Drug-likeness (DL) is a qualitative concept estimating the druggability of compounds, which assesses the compounds' capability of modulating targets [23]. Based on the TCMSP database, OB $\geqq 30 \%$, IEP $\geqq-0.4$, and $\mathrm{DL} \geqq 0.18$ were set as the pharmacokinetic criteria to screen out the bioactive compounds of DNt [24]. The molecule structures of all the bioactive compounds were identified using the PubChem database [25] (https://pubchem.ncbi. nlm.nih.gov/).

2.2. Putative Targets of DNt. The putative targets corresponding to the bioactive compounds of DNt were collected from three databases: (1) TCMSP database provides the related targets for the certain compounds; (2) Swiss Target Prediction [26] platform (http://www.swisstargetprediction.ch) predicts targets that have the actives displaying similarity with the query molecule. The species is limited to "Homo sapiens," and only the results with a probability value of $>0.7$ were collected. (3) PharmMapper [27] platform (http://lilab-ecust.cn/pharmmapper/submitfile.html) predicts targets via a pharmacophore mapping approach; the targets with the setting of "human protein targets only" and a fit score of $>3.5$ were obtained. After eliminating duplicates, all the predicted targets were converted into Uniprot IDs using UniProtKB database (https://www.uniprot.org/) for the subsequent study.

2.3. Therapeutic Targets of DNt against NAFLD. Four disease databases, MalaCards [28] (https://www.malacards.org), DisGeNET [29] (http://www.disgenet.org/), Comparative Toxicogenomics Database [30] (CTD, http://ctdbase.org), and Online Mendelian Inheritance in $\operatorname{Man}^{\circledR}$ (OMIM) [31] database (http://www.omim.org/) were used to collect the NAFLD-related targets. The search terms used were "nonalcoholic fatty liver disease" or "NAFLD".

Based on the preceding steps, two sets of targets had been prepared: the putative targets of DNt and the NAFLD-related targets. Venn diagram package in $R$ was used to screen the 


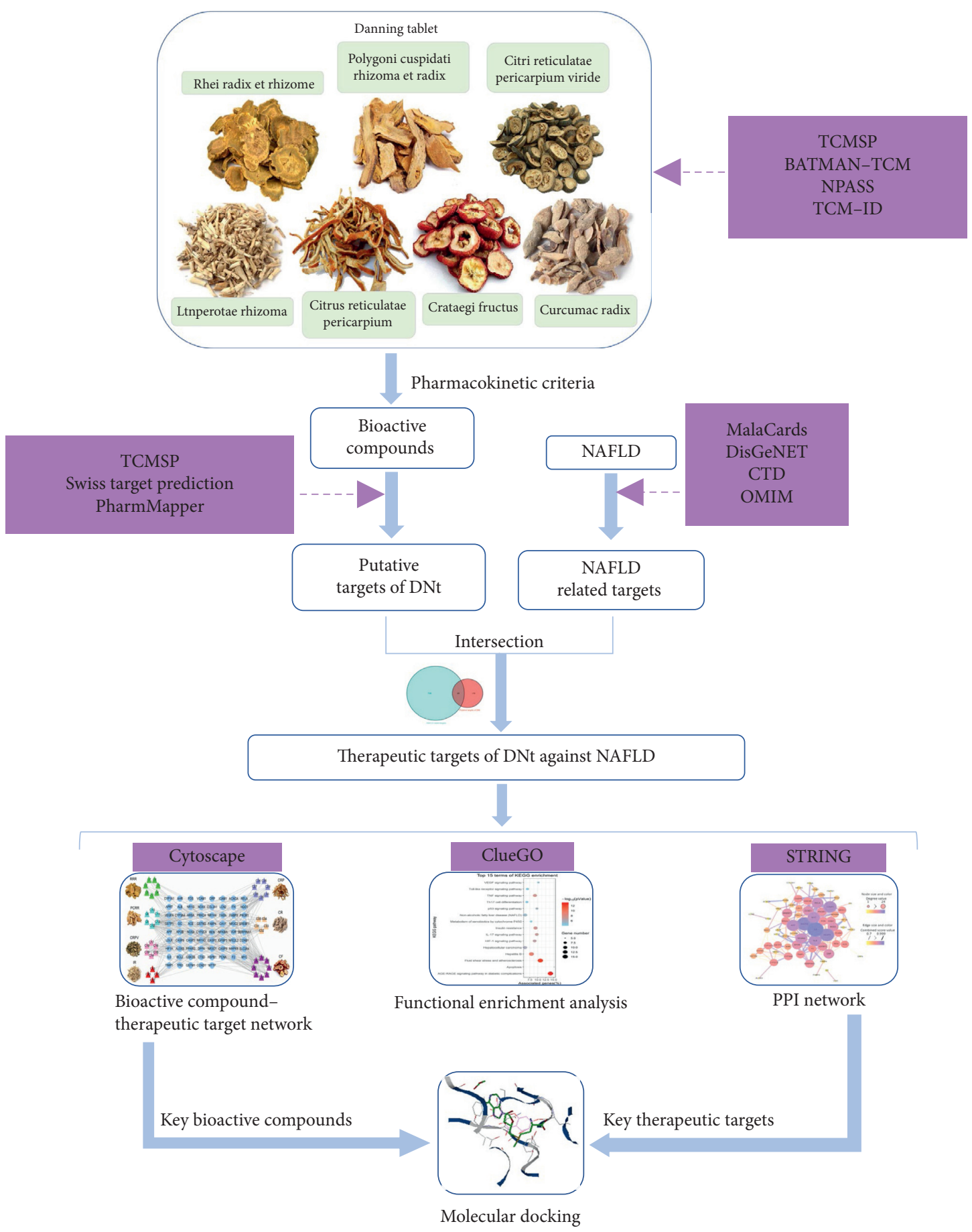

Figure 1: A flowchart of this study.

overlapping targets, which were identified as the potential therapeutic targets of DNt against NAFLD.

2.4. Network Construction and Analysis. Two networks were constructed in this study: (1) a bioactive compound-therapeutic target network was built to show the interactions of the bioactive compounds and the therapeutic targets of DNt; (2) a protein-protein interaction (PPI) network was built to explore the interactions of the therapeutic targets of $\mathrm{DNt}$ against NAFLD. The PPI analysis was conducted using Search Tool for the Retrieval of Interacting Genes/Proteins platform [32] (STRING, https://string-db.org/), and the criteria were limited in "Homo sapiens" and a high confidence score of 0.7 .

The networks were constructed by Cytoscape software version 3.7.2 [33], and the NetworkAnalyzer plug-in was used to analyze the nodes' topological parameters in the network. Two topological parameters, degree and closeness centrality, were calculated to measure the importance of the 
nodes. A degree value is defined as the number of edges linked to a node. A closeness centrality value is defined as the average of the shortest path length from a node to all other nodes, indicating the closeness of a node to the others in the network [34]. The key compounds and targets were screened out according to their network topological property, which was defined as two times greater than the median degree value and one time greater than the median closeness centrality value of all nodes in the network [35].

2.5. Functional Enrichment Analysis. To elucidate the biological mechanisms of therapeutic targets of DNt acting on NAFLD on a systematic level, the ClueGO 2.5.4 plug-in of Cytoscape was used to perform Gene Ontology (GO) and Kyoto Encyclopedia of Genes and Genomes (KEGG) pathway functional enrichment analysis. The minimum gene counts enriched in each GO or KEGG term was set as three, and the terms with $p$ values $<0.05$ were considered statistically significant. The "ggplot2" package in $R$ software (version 4.0.3, the $R$ Foundation for Statistical Computing, Vienna, Austria) was used to visualize the GO and KEGG terms.

2.6. Molecular Docking Simulation. To evaluate the binding potential of the key bioactive compounds of DNt to key target proteins, molecular docking simulation was performed using GEMDOCK software (version 2.1, Hsinchu, Taiwan) [34]. The empirical scoring function of GEMDOCK is as follows: Fitness = van der Waal energy $(\mathrm{vdW})+$ hydrogen bonding energy (Hbond) + electro statistic energy (Elec). A fitness value was used to estimate the binding affinity of a protein-ligand complex. A lower fitness value indicates a stabler binding between a protein and a ligand. The fitness value of the corresponding protein-original ligand was used as a comparison. And the generic evolutionary method parameters were set as population size $=200$, generations $=70$, and number of solutions $=2$.

All 3D crystal structures of the target protein-ligand complexes were retrieved from Protein Data Bank [35] (PDB, http://www.rcsb.org/pdb/). All 3D molecular structures of the compounds were retrieved from ZINC15 database [36] (http://zinc.docking.org).

\section{Results}

3.1. Bioactive Compounds and Putative Targets of DNt. A total of 1303 compounds of DNt were collected from four databases, including 239 compounds in RRR, 188 compounds in PCRR, 54 compounds in CRPV, 64 compounds in IR, 348 compounds in CRP, 315 compounds in CR, and 95 compounds in CF. After excluding duplicates, we finally screened out 43 bioactive compounds with eligible OB, IEP, and DL properties (shown in Supplementary Table 1). What is more, 219 diverse putative targets of these bioactive compounds were obtained from the three target prediction databases.
3.2. Bioactive Compound-Therapeutic Target Network. We collected a total of 779 NAFLD-related targets from the four databases after the removal of duplicates. And 69 overlapping targets between the putative targets of DNt and NAFLD-related targets were recognized as the therapeutic targets of DNt in the treatment of NAFLD (Figure 2(a) and Supplementary Table 2).

The bioactive compound-therapeutic target network (Figure 2(b)) consisted of 112 nodes (43 bioactive compounds and 69 therapeutic targets) and 356 edges, manifesting a multicompound and multitarget mode of action. Seven bioactive compounds with the highest degree values, quercetin $($ degree $=41), \quad$ luteolin $\quad($ degree $=20), \quad \beta$-sitosterol $($ degree $=18)$, curcumenolactone $C($ degree $=17)$, supraene $($ degree $=17)$, kaempferol $($ degree $=18)$, and stigmasterol (degree $=16)$ were recognized as the key bioactive compounds of DNt against NAFLD, owing to that they were twofold higher than the median degree value of 7 and onefold higher than the median closeness centrality value of 0.37 (Table 1). Higher degree values indicate that these bioactive compounds interact with more therapeutic targets, and higher closeness centrality values indicate their more central position in the network. Additionally, six of the bioactive compounds were from CF, three of them were from PCRR, manifesting the contributions of CF and PCRR in DNt.

3.3. Functional Enrichment Analysis. The functional enrichment analysis was performed for the 69 therapeutic targets. In total, 359 GO terms were identified, including 35 molecule function (MF) terms, three cellular component (CC) terms, and 320 biological process (BP) terms. For BP, the therapeutic targets were mainly responsible for the responses to oxidative stress, xenobiotic stimulus, inflammation, vitamin $\mathrm{D}$, and the regulation of lipid, apoptosis, and foam cell differentiation. For $\mathrm{MF}$, the therapeutic targets mainly participated in the binding of protease, fatty acid, steroid, oxygen, glutathione, and the activation of the transcription factor, vitamin D 24-hydroxylase, and cysteine-type endopeptidase involved in apoptosis. For CC, the potential targets were mainly distributed in the endoplasmic reticulum (ER), death-inducing signaling complex, and platelet. The most significantly enriched 15 GO terms are visualized in Figure 3(a)-3(c).

Sixty-one KEGG pathway terms were enriched (Figure 3(d)); among them, signaling pathways of apoptosis, advanced glycation end products (AGE) and receptor for AGE (RAGE), and fluid shear stress and atherosclerosis had the highest proportion of the target genes. The top 15 most significantly enriched terms are shown in Figure 3(e).

3.4. PPI Network of the Therapeutic Targets. Because two targets were excluded for the lack of any interaction with other proteins, the PPI network consisted of 235 interactions among 67 therapeutic targets (Figure 4). The median degree value and median closeness centrality value of the PPI network were 6 and 0.31 , respectively. Nine proteins, interleukin-6 (IL6), mitogen-activated protein kinase 8 (MAPK8), vascular endothelial growth factor A (VEGFA), caspase-3 (CASP3), Myc proto-oncogene protein (MYC), 


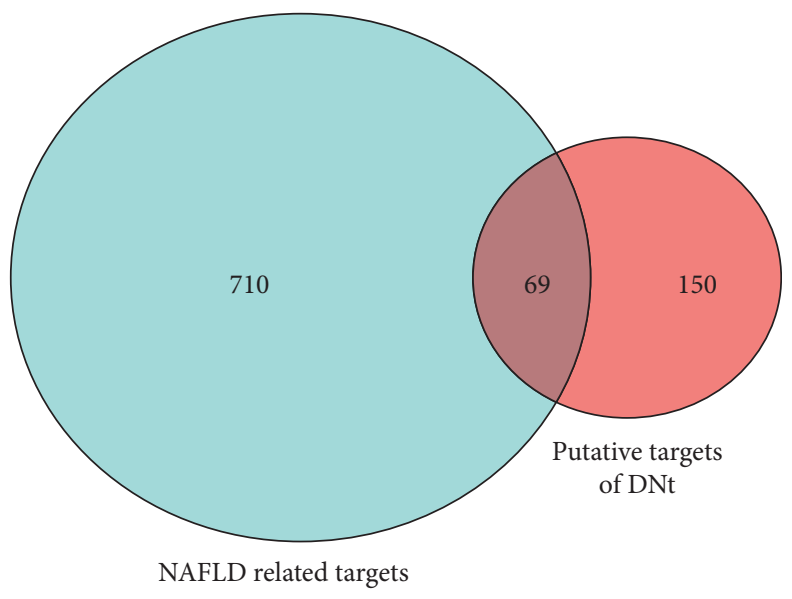

(a)
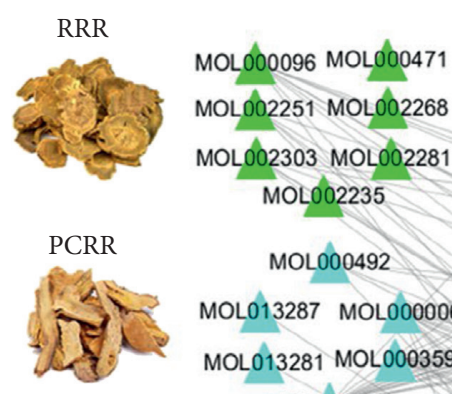

MOL000492

CYP1A1 AHR FOS VCAM

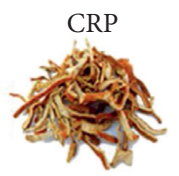
MOL013287 MOLO00006
MOL013281 MOL000359
MOLO00098

APRT ALB NR1I3 NOX4 $\mathrm{COL3A1}$ LCN2 ITK NQO1

VEGFA CYP3A4 ARSA PRKCA NR1H4 FASN FABP3 PIK3R1

GSTP1 GC ACE GSTM2 FABP4 CAV1 NR3C2 SREBF1

MOL004306 MOL004263

Motolos

APP APOB NOS3 GYP2C9 REN NFKBIA VDR SERPINA1 MOL004253

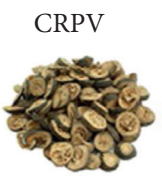

MOL001803
MOL005828 MOL005100

LDLR CASP8 CASP1 NR1H3 CASP3 IGFBP3 NFE2L2 CCNB1

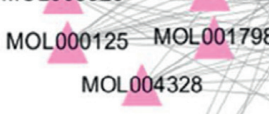

HIF1A ALOX5 PPARG DPP4 NR3C1 CASP9 MAPK8 SLC2A4 MOLOO 3578
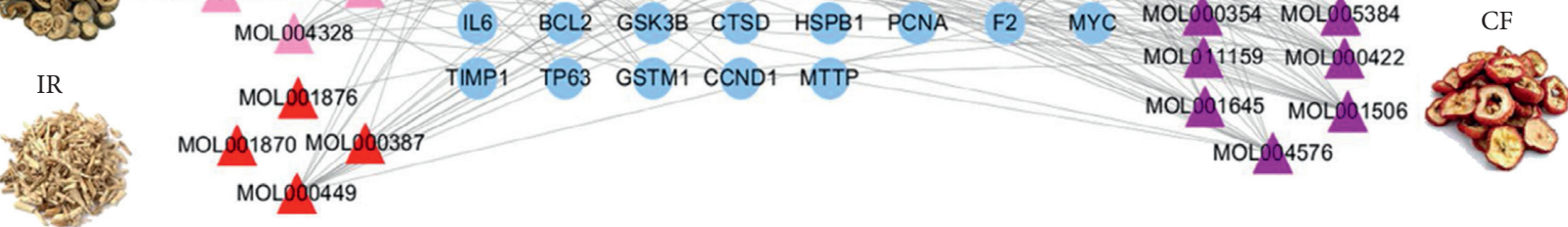

(b)

Figure 2: The therapeutic targets of DNt against NAFLD and the bioactive compound-therapeutic target network. (a) The Venn diagram of the overlapping targets between the putative targets of DNt and NAFLD-related targets. (b) The bioactive compound-therapeutic target network of DNt against NAFLD. Triangle nodes represent the bioactive compounds of DNt; circular nodes represent the therapeutic targets; edges represent the relations between compounds and targets. RRR, Rhei Radix et Rhizome; PCRR, Polygoni Cuspidati Rhizoma et Radix; CRPV, Citri Reticulatae Pericarpium Viride; IR, Imperatae Rhizoma; CRP, Citrus Reticulatae Pericarpium; CR, Curcumae Radix; CF, Crataegi Fructus.

serum albumin (ALB), amyloid-beta A4 protein (APP), peroxisome proliferator-activated receptor gamma (PPARG), and transcription factor p65 (RELA) met the screening criteria of key nodes in this network, demonstrating their central roles in the pathological processes of NAFLD. These targets acted as irreplaceable mediums to establish the connections between other targets in the development of NAFLD, so they were identified as the key targets of DNt treating NAFLD. Table 2 lists the detailed information of the key therapeutic targets.

3.5. Molecular Docking Simulation. Nine key target proteins, IL6, MAPK8, VEGFA, CASP3, MYC, APP, ALB, PPARG, and RELA, were docked with seven key bioactive compounds, quercetin, luteolin, $\beta$-sitosterol, curcumenolactone $\mathrm{C}$, supraene, kaempferol, and stigmasterol. The results found that 30 target protein-bioactive compound complexes (47.62\% of all) might bind firmer than the corresponding protein-original ligand complexes, indicating that numerous key bioactive compounds might bind closely with the key therapeutic targets to exercise regulatory effects. Among all, the RELA-quercetin complex had the best fitness value of $-113.07 \mathrm{Kcal} / \mathrm{mol}$, followed by ALB-stigmasterol $(-111.65 \mathrm{Kcal} / \mathrm{mol})$ and RELA-luteolin $(-110.22 \mathrm{Kcal} / \mathrm{mol})$. Table 3 shows the results of docking, and the docking model of every certain key target and the corresponding bestbinding bioactive compound is shown in Figure 5. 
TABLE 1: The information of the key bioactive compounds of DNt.

\begin{tabular}{|c|c|c|c|c|c|c|c|c|c|}
\hline TCMSP ID & Name & $\begin{array}{l}\text { Molecular } \\
\text { formula }\end{array}$ & $\begin{array}{c}\text { Structural } \\
\text { formula }\end{array}$ & $\begin{array}{l}\text { OB } \\
(\%)\end{array}$ & IEP & $\mathrm{DL}$ & Source & $\begin{array}{c}\text { Degree } \\
\text { value }\end{array}$ & $\begin{array}{c}\text { Closeness } \\
\text { centrality value }\end{array}$ \\
\hline MOL000098 & Quercetin & $\mathrm{C}_{15} \mathrm{H}_{10} \mathrm{O}_{7}$ & & 46.43 & 0.05 & 0.28 & PCRR, CF & 41 & 0.53 \\
\hline MOL000006 & Luteolin & $\mathrm{C}_{15} \mathrm{H}_{10} \mathrm{O}_{6}$ & & 36.16 & 0.19 & 0.25 & PCRR, CF & 20 & 0.43 \\
\hline MOL000359 & $\beta$-sitosterol & $\mathrm{C}_{29} \mathrm{H}_{50} \mathrm{O}$ & & 36.91 & 1.32 & 0.75 & $\begin{array}{c}\text { PCRR, IR, CRP, } \\
\text { RRR, CR, CF }\end{array}$ & 18 & 0.43 \\
\hline MOL004253 & $\begin{array}{c}\text { Curcumenolactone } \\
\text { C }\end{array}$ & $\mathrm{C}_{15} \mathrm{H}_{20} \mathrm{O}_{4}$ & & 39.7 & 0.16 & 0.19 & $\mathrm{CR}$ & 17 & 0.43 \\
\hline MOL001506 & Supraene & $\mathrm{C}_{30} \mathrm{H}_{50}$ & & 33.55 & 2.08 & 0.42 & $\mathrm{CF}$ & 17 & 0.41 \\
\hline MOL000422 & Kaempferol & $\mathrm{C}_{15} \mathrm{H}_{10} \mathrm{O}_{6}$ & & 41.88 & 0.26 & 0.24 & $\mathrm{CF}$ & 17 & 0.40 \\
\hline MOL000449 & Stigmasterol & $\mathrm{C}_{29} \mathrm{H}_{48} \mathrm{O}$ & & 43.83 & 1.44 & 0.76 & $\mathrm{IR}, \mathrm{CF}$ & 16 & 0.42 \\
\hline
\end{tabular}

OB, oral bioavailability; IEP, intestinal epithelial permeability; DL, drug-likeness.

\section{Discussion}

In the present study, the multicompound, multitarget, multipathway mechanisms of action of DNt against NAFLD were clarified applying the network pharmacology approach.

First of all, the bioactive compounds and the putative targets of DNt were identified, and the overlapping targets between DNt and NAFLD were recognized as the therapeutic targets of DNt against NAFLD (Figure 2(a)). Quercetin, $\beta$-sitosterol, luteolin, kaempferol, supraene, curcumenolactone $\mathrm{C}$, and stigmasterol were selected as the key bioactive compounds of DNt treating NAFLD, based on their contributions in the bioactive compound-therapeutic target network (Figure 2(b) and Table 1). Quercetin, luteolin, and kaempferol are all members of the flavone family, which are widely found in vegetables, fruits, and herbs. The flavones display extensive pharmacological activities, including anti-inflammatory, antioxidative, antimicrobial, antiapoptotic, hepatoprotective, and anticancer [37]. Quercetin could ameliorate NAFLD progression by mitigating oxidative stress, inflammation, and lipid metabolic disorders [38]. It could also modulate the intestinal probiotics and sequentially regulate gut-liver axis activation in NAFLD [39]. Luteolin could relieve diet-induced obesity and its metabolic complications, such as adiposopathy, hepatic steatosis, and IR, by regulating PPARG [40] and Toll-like receptor signaling pathways [41]. Luteolin and kaempferol could both abrogate lipid accumulation induced by the activation of liver $X$ receptor- (LXR-) sterol regulatory element-binding protein 1c (SREBP-1c) pathway to mitigate hyperlipemia, NAFLD, and other components of metabolic syndrome (MetS) [42-44].

Supraene, also known as squalene, is a triterpenoid performing anticancer, antioxidant, and detoxicant bioactivities. Supraene had anticholesterolemia and antiatherosclerotic effects through the transactivation of LXR but without the alteration of SREBP-1c expression [45]. $\beta$-sitosterol and stigmasterol are two kinds of the most occurring phytosterols, which can decrease the absorption of dietary lipids and bile acids and attenuate hepatic lipid accumulation and weight gain $[46,47]$. Curcumenolactone is a carabrane-type sesquiterpene lactones. A previous study showed curcumenolactones A, B, C could protect the liver from D-galactosamine-induced acute injury in mice [48]. To conclude, three flavonoids (quercetin, luteolin, and kaempferol), three natural lipids ( $\beta$-sitosterol, supraene, stigmasterol), and one sesquiterpene lactone undertake the pharmacology basis of the anti-NAFLD effects of DNt, which were consistent with the pathogenesis of promotion of NAFLD.

When it comes to herbs, CF and PCRR stood out for their major contributions of providing more bioactive compounds. In a systematic review, CF was the most frequently used herb in the treatment of NAFLD [49], the extract of which could significantly enhance the activities of antioxidant enzymes, lipid metabolism enzymes, and fatty acid oxidation-related enzymes in the liver of high-fat dietfed mice [50]. The PCRR extract could inhibit cell proliferation, cell migration, and vessel formation in endothelial cells by suppressing VEGF-induced activations of c-Jun 


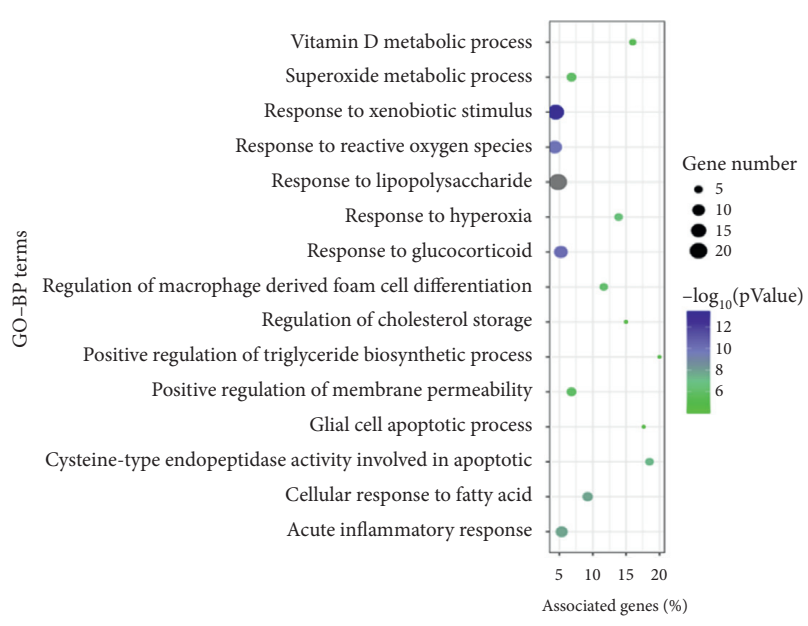

(a)

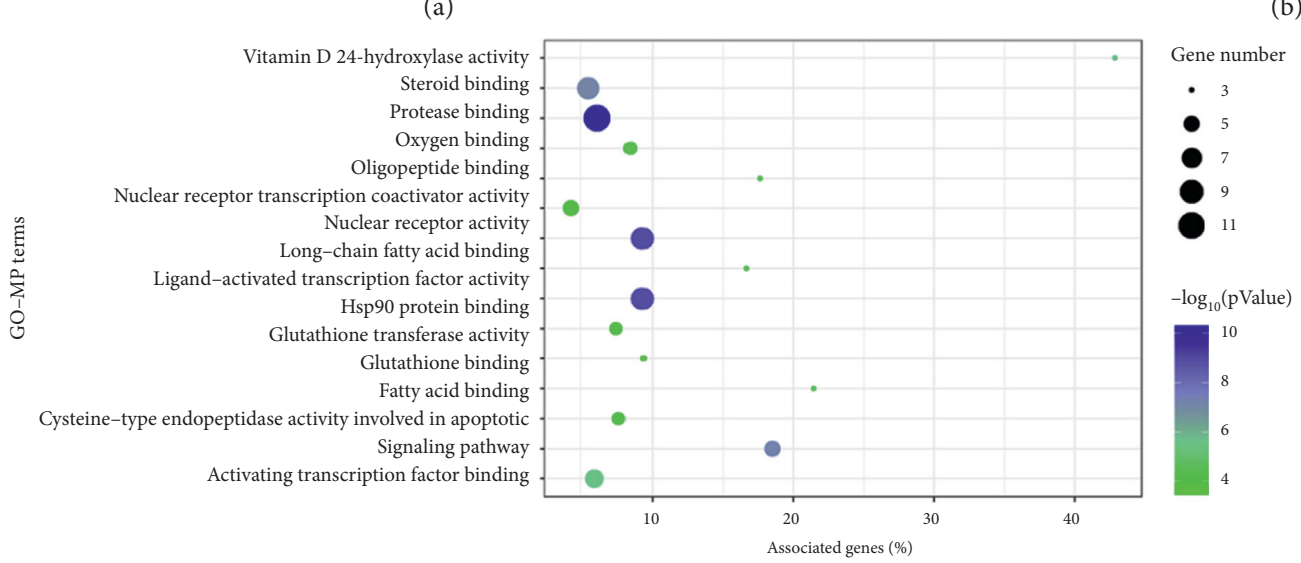

(c)

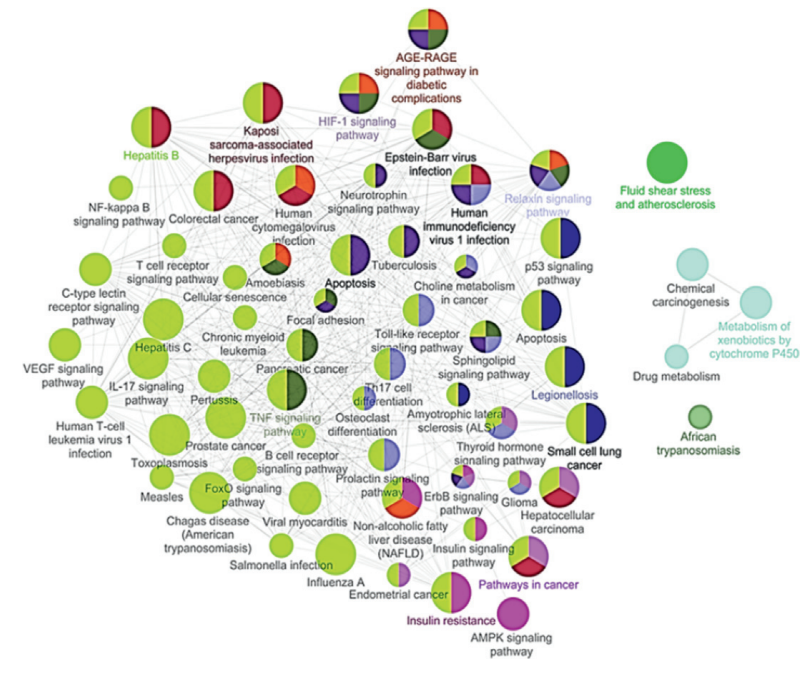

(d)

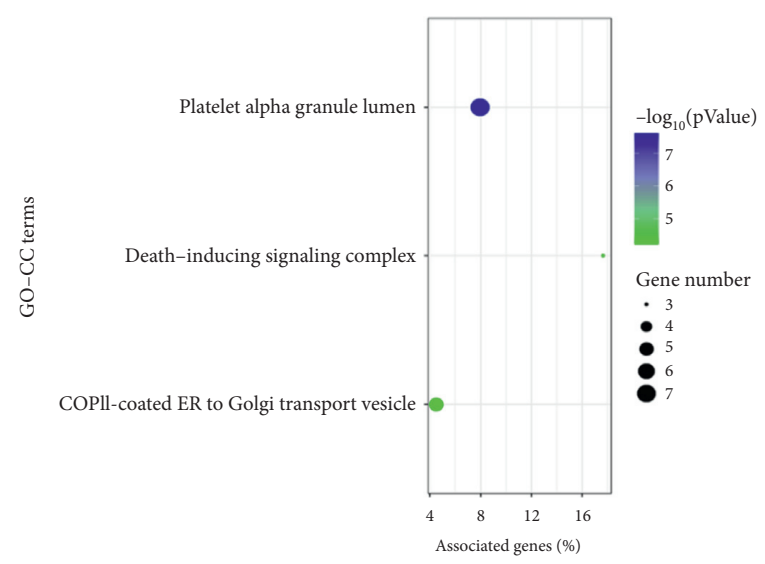

(b)

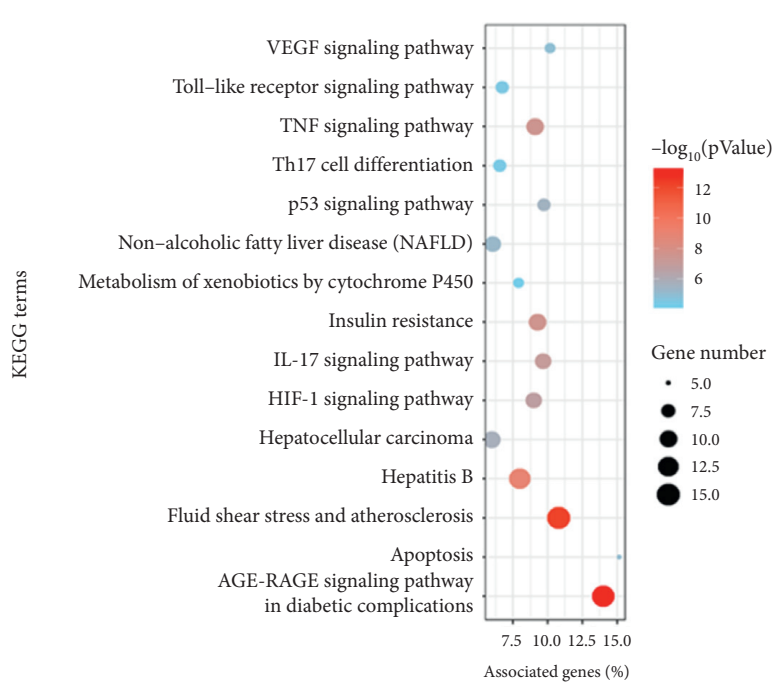

(e)

FIgURE 3: Functional enrichment analysis of the therapeutic targets of DNt against NAFLD. (a) The top 15 GO-BP terms; (b) all the GO-CC terms; (c) the top 15 GO-MF terms. (d) The KEGG pathway enrichment conducted by ClueGO: nodes represent KEGG terms, the node's size represents the enriched significance, and the node's color reflects the enriched classification. (e) The top 15 KEGG pathway terms. Note: the bubble size represents the count of therapeutic targets enriched in a certain pathway; the associated genes (\%) mean the percentage of target genes to the background genes of a certain pathway. GO, Gene Ontology; BP, biological process; CC, cellular component; MF, molecule function; KEGG, Kyoto Encyclopedia of Genes and Genomes. 


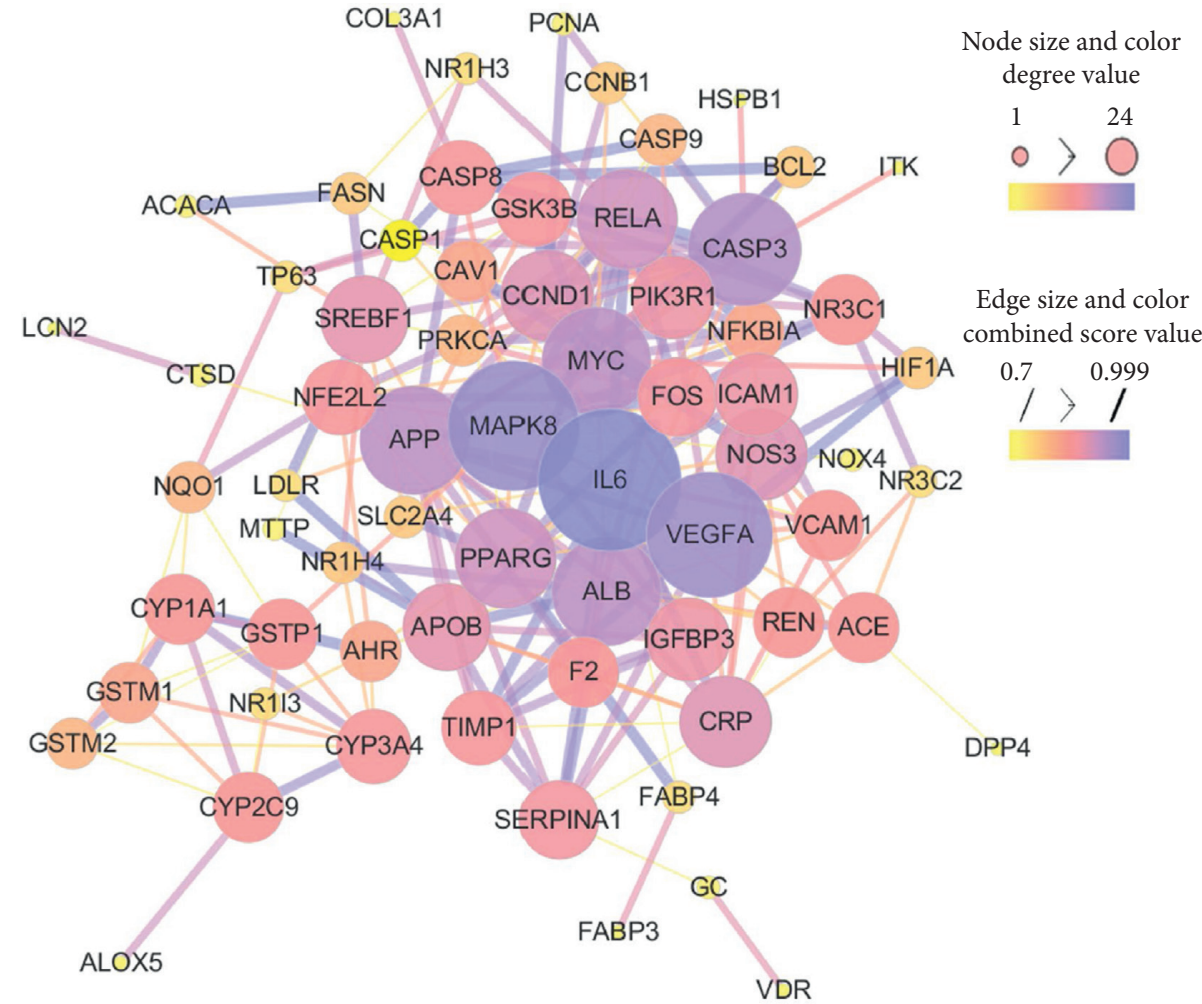

FIGURE 4: The PPI network of the therapeutic targets of DNt against NAFLD. The size and color of a node are proportional to the degree value, the size and color of an edge are proportional to the combined score. Smaller sizes and brighter colors signify lower degree values.

TABLE 2: The key therapeutic targets of DNt in the treatment of NAFLD.

\begin{tabular}{lccccc}
\hline No. & Target name & Gene symbol & Uniprot ID & Degree value & Closeness centrality value \\
\hline 1 & Interleukin-6 & IL6 & P05231 & 24 & 0.55 \\
2 & Mitogen-activated protein kinase 8 & MAPK8 & P45983 & 21 & 0.53 \\
3 & Vascular endothelial growth factor A & VEGFA & P15692 & 20 & 0.52 \\
4 & Caspase-3 & CASP3 & P42574 & 17 & 0.50 \\
5 & Myc proto-oncogene protein & MYC & P01106 & 16 & 0.49 \\
6 & Amyloid-beta A4 protein & APP & P05067 & 16 & 0.50 \\
7 & Serum albumin & ALB & P02768 & 16 & 0.48 \\
8 & Peroxisome proliferator-activated receptor gamma & PPARG & P37231 & 14 & 0.47 \\
9 & Transcription factor p65 & RELA & Q04206 & 14 & 0.47 \\
\hline
\end{tabular}

$\mathrm{N}$-terminal kinase (JNK) and reactive oxygen species (ROS) [51]. As for the other component herbs, the citrus flavonoids isolated from CRP might exert hepatoprotective and antiinflammatory effects by suppressing nuclear factor- $\kappa \mathrm{B}(\mathrm{NF}-$ $\kappa \mathrm{B})$ and MAPK signaling pathways in rats with NAFLD [52]. RRR was reported to show liver-protective function [53]; the extract of IR showed antioxidation ability in vitro [54]; the combined treatment with CR and Glycyrrhizae Radix et Rhizoma could inhibit body weight gain, lipid metabolic disturbances, serum liver enzymes, and hepatic steatosis in rats [55].

Then, the functional enrichment analysis of the therapeutic targets demonstrated the mechanisms of DNt against NAFLD might be focused on apoptosis-associated pathways, MetS-associated pathways (e.g., AGE-RAGE, IR, and atherosclerosis pathways), inflammation-associated pathways (e.g., IL-17, TNF, hypoxia-inducible factor-1 (HIF-1), and
Toll-like pathways), and cancer-associated pathways (e.g., VEGF and p53 pathways) (Figure 3). As components of MetS, NAFLD and atherosclerosis share various pathophysiological mechanisms, including inflammation, oxidative stress, unbalanced coagulation-fibrinolysis, chronic intermittent hypoxia, altered adipokine profile, and unfavorable lipidosis [56]. AGEs accelerate the production of RAGEs and the subsequent AGE-RAGE interactions contribute to hepatic fat accumulation, giving rise to inflammation, IR, fibrosis, and other complications of NAFLD [57].

After that, IL6, MAPK8, VEGFA, CASP3, MYC, APP, ALB, PPARG, and RELA were identified as the key targets of DNt against NAFLD, due to their high degree and closeness centrality values (Figure 4). These targets interacted broadly and closely with other targets, indicating their central roles in the disease network. IL-6 has been acknowledged as a 
TABLE 3: The results of molecular docking simulation.

\begin{tabular}{|c|c|c|c|c|c|}
\hline Protein (PDB ID) & Ligand & Fitness $(\mathrm{Kcal} / \mathrm{mol})$ & Protein (PDB ID) & Ligand & Fitness $(\mathrm{Kcal} / \mathrm{mol})$ \\
\hline \multirow{8}{*}{ IL6 (5FUC) } & $\mathrm{NAG}^{*}$ & -82.47 & \multirow{8}{*}{ MAPK8 (2GMX) } & $877^{*}$ & -105.32 \\
\hline & Quercetin & -96.46 & & Stigmasterol & -103.52 \\
\hline & Kaempferol & -94.28 & & $\beta$-Sitosterol & -102.45 \\
\hline & Supraene & -93.65 & & Supraene & -98.66 \\
\hline & Luteolin & -86.82 & & Luteolin & -90.16 \\
\hline & Stigmasterol & -83.16 & & Curcumenolactone C & -88.64 \\
\hline & Curcumenolactone $\mathrm{C}$ & -78.64 & & Quercetin & -80.01 \\
\hline & $\beta$-Sitosterol & -78.38 & & Kaempferol & -78.28 \\
\hline \multirow{8}{*}{ VEGFA (6D3O) } & $\mathrm{XCP}^{*}$ & -74.42 & \multirow{8}{*}{ CSP3 (3GJR) } & DZE* $^{*}$ & -64.24 \\
\hline & Quercetin & -81.67 & & Luteolin & -109.76 \\
\hline & Kaempferol & -81.48 & & Quercetin & -101.75 \\
\hline & Curcumenolactone C & -65.03 & & $\beta$-Sitosterol & -93.91 \\
\hline & Supraene & -62.96 & & Kaempferol & -92.97 \\
\hline & Luteolin & -62.85 & & Stigmasterol & -90.54 \\
\hline & Stigmasterol & -53.81 & & Supraene & -85.62 \\
\hline & $\beta$-Sitosterol & -51.30 & & Curcumenolactone C & -82.71 \\
\hline \multirow{8}{*}{ MYC (5I4Z) } & $\mathrm{GOL}^{*}$ & $\mathrm{v} 42.74$ & \multirow{8}{*}{ APP (3OVJ) } & ORA* & -89.52 \\
\hline & Supraene & -102.18 & & Stigmasterol & -82.77 \\
\hline & $\beta$-Sitosterol & -89.16 & & $\beta$-Sitosterol & -80.19 \\
\hline & Stigmasterol & -85.25 & & Supraene & -76.58 \\
\hline & Luteolin & -83.55 & & Quercetin & -69.45 \\
\hline & Quercetin & -78.06 & & Luteolin & -68.01 \\
\hline & Kaempferol & -73.66 & & Kaempferol & -66.05 \\
\hline & Curcumenolactone C & -71.46 & & Curcumenolactone C & -59.21 \\
\hline \multirow{8}{*}{ ALB (1E7A) } & PFL $^{*}$ & -69.57 & \multirow{8}{*}{ PPARG (2ATH) } & $3 \mathrm{EA}^{*}$ & -97.19 \\
\hline & Stigmasterol & -111.65 & & Luteolin & -99.33 \\
\hline & Luteolin & -107.49 & & Stigmasterol & -97.79 \\
\hline & $\beta$-Sitosterol & -100.86 & & $\beta$-Sitosterol & -95.81 \\
\hline & Curcumenolactone C & -93.57 & & Supraene & -93.44 \\
\hline & Supraene & -90.49 & & Kaempferol & -93.44 \\
\hline & Quercetin & -88.94 & & Quercetin & -91.09 \\
\hline & Kaempferol & -86.36 & & Curcumenolactone C & -83.09 \\
\hline \multirow{4}{*}{ RELA (3QXY) } & SAM $^{*}$ & -142.70 & \multirow{4}{*}{ RELA (3QXY) } & Kaempferol & -107.02 \\
\hline & Quercetin & -113.07 & & $\beta$-Sitosterol & -95.78 \\
\hline & Luteolin & -110.22 & & Stigmasterol & -94.77 \\
\hline & Supraene & -107.85 & & Curcumenolactone C & -83.78 \\
\hline
\end{tabular}

${ }^{*} \mathrm{NAG}, 877$, XCP, DZE, GOL, ORA, PFL, 3EA, and SAM are the names of original ligands for corresponding targets in the PDB database.

central factor in liver inflammation. The activation of IL-6 signaling pathway can induce the production of acute-phase proteins, such as fibrinogen and haptoglobin, leading to hepatitis syndrome, cholestasis subtype, fibrosis, and worse [58]. The activation of MAPK8, also named JNK1, participates in fat-induced apoptosis and IR, and the latter would propel NAFLD to more advanced stages [59]. VEGFA is a sentinel regulator of angiogenesis. It is widely believed that angiogenesis is correlated with NAFLD and fibrogenic progression [60]. For example, serum VEGFA and VEGFR1 levels were significantly elevated in NAFLD patients, and the borderline was even higher in NASH patients [61].

Among the key targets, CASP3 is well-known for its irreplaceable role in the apoptosis process, which can promote inflammation, IR, fibrosis, and cirrhosis in turn [62]. RELA is one protein of the NF- $\kappa$ B family. Saturated fatty acids can activate $\mathrm{NF}-\kappa \mathrm{B}$ and MYC signaling pathways, followed by increased proinflammatory cytokines, such as TNF- $\alpha$ and IL-6, resulting in the development of IR and NAFLD $[63,64]$. APP is the precursor protein of amyloid- $\beta$, which is a consequence of IR and is widely known as a key pathological hallmark of Alzheimer's disease [65]. Moreover, APP can also promote IR in the liver in turn [66]. ALB acts as a key carrier in plasma, possessing the ability of binding with free fatty acids, inflammatory mediators, ROS, lipopolysaccharide, and bacterial antigens, which enable it to perform a vast series of regulatory activity in inflammation, antioxidation, and endothelial function [67, 68]. PPARG is one isotype of PPARs, which is pivotal for the lipogenesis in hepatocytes. Therefore, PPARG is regarded as a characteristic of NAFLD [69]. Contradictorily, PPARG also possesses anti-inflammatory properties, especially in macrophages [70], which might limit hepatic stellate cell proliferation and subsequent fibrosis [71]. Thus, it is undoubted that PPARG plays a critical role in NAFLD, but its multiple actions are still controversial. To sum up, it was speculated that $\mathrm{DNt}$ might perform integrative functions of lipid mediation, antiinflammation, antioxidant, anti-IR, antiapoptosis, and antiendothelial/fibrosis on NAFLD through key targets in associated signaling pathways. 


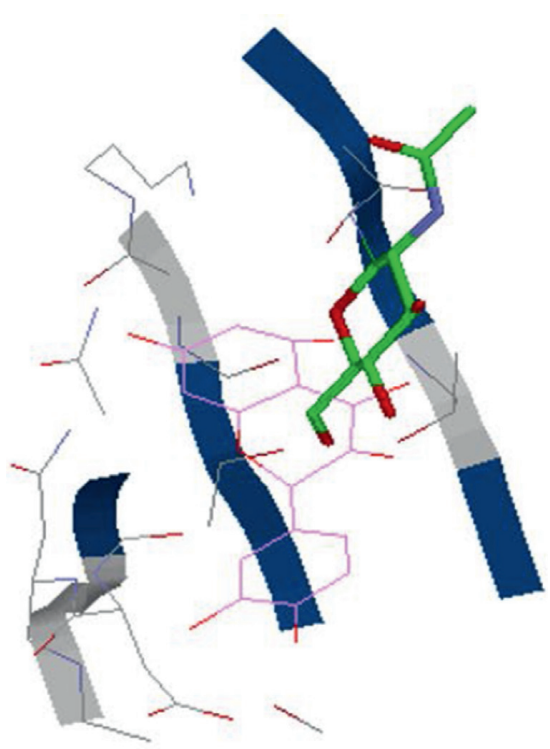

(a)

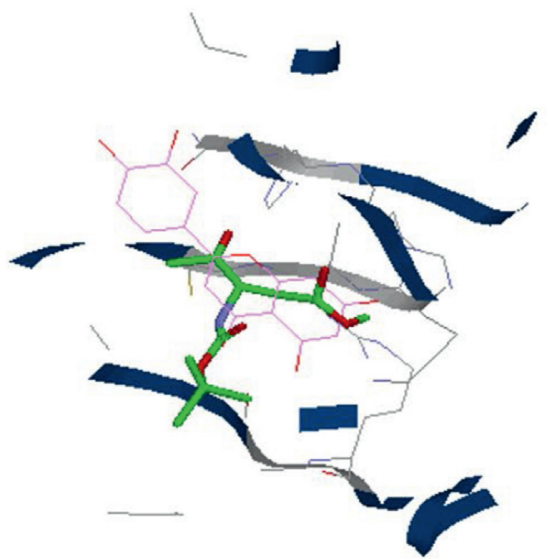

(d)

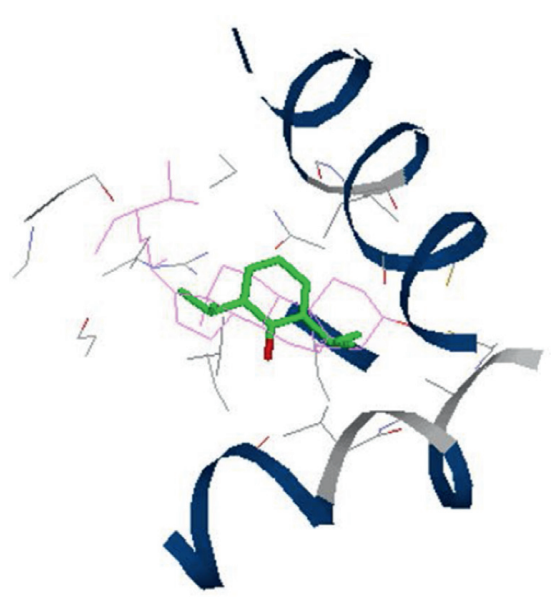

$(\mathrm{g})$

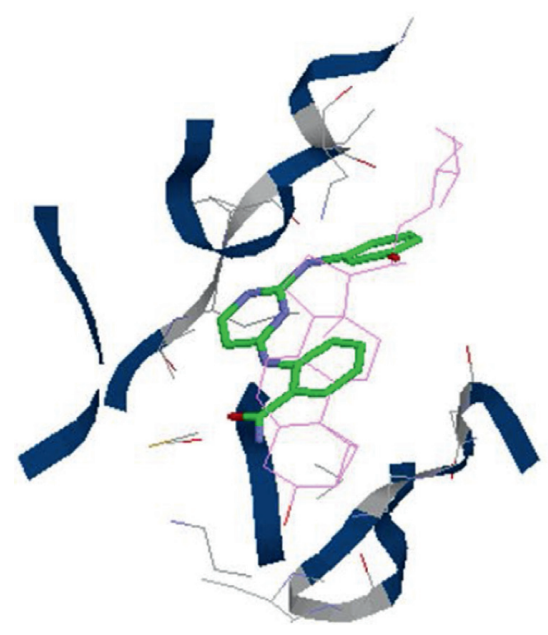

(b)

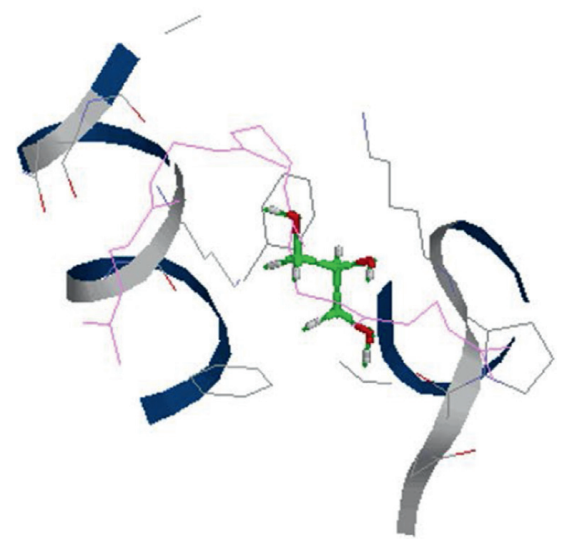

(e)

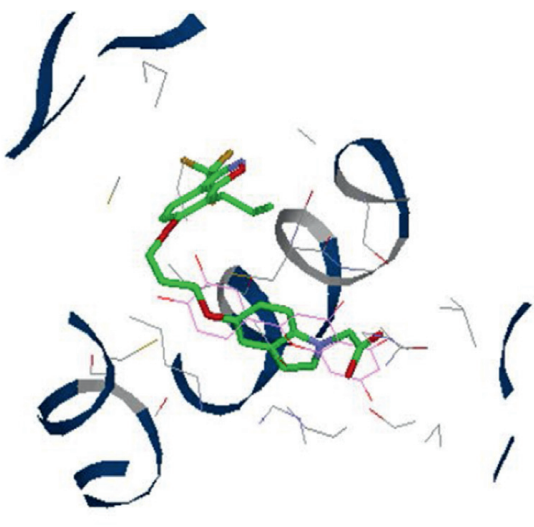

(h)

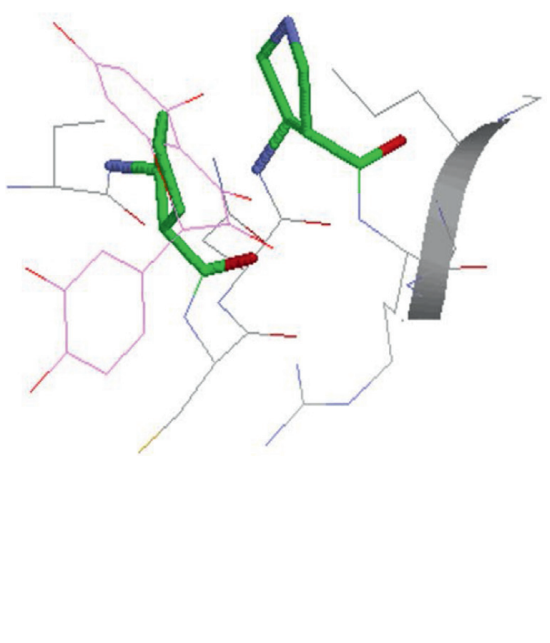

(c)

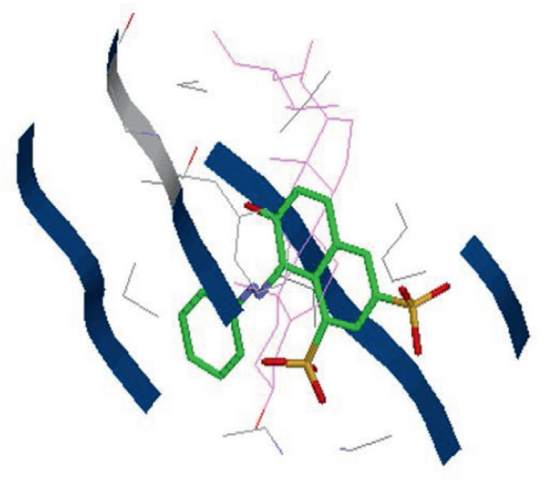

(f)

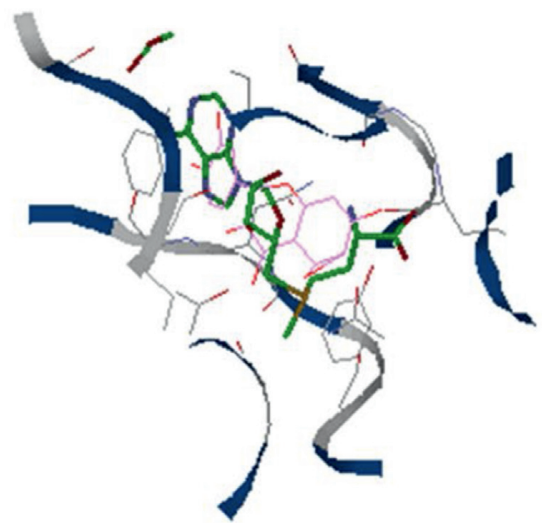

(i)

FIGURE 5: Molecular docking models of the key target proteins with the key bioactive compounds. (a) IL6-quercetin, (b) MAPK8-stigmasterol, (c) VEGFA-quercetin, (d) CSP3-luteolin, (e) MYC-supraene, (f) APP-stigmasterol, (g) ALB-stigmasterol, (h) PPARG-luteolin, and (i) RELAquercetin. The original ligands are labeled in green color, and the predicted poses of the bioactive compounds are labeled in pink color. 
In the end, the molecular docking (Table 3 and Figure 5) showed that about half of the key bioactive compounds might bind firmly to the key target proteins, which provided the possibility of compound-target combination and pharmacological activities realization. The molecular docking simulation should strengthen the ratiocinations in this study, to some extent, but experimental validations are still needed.

\section{Conclusions}

This study applied a network pharmacology approach to elucidate the underlying mechanisms of DNt against NAFLD in a holistic manner. Seven bioactive compounds of $\mathrm{DNt}$, quercetin, $\beta$-sitosterol, luteolin, kaempferol, supraene, curcumenolactone $\mathrm{C}$, and stigmasterol, might exert the crucial pharmacological effects. Moreover, IL6, MAPK8, VEGFA, CASP3, MYC, APP, ALB, PPARG, and RELA might be the key targets regulated by those bioactive compounds. Besides, signaling pathways of apoptosis, inflammation, oxidant stress, IR, and lipid metabolism should be mainly involved. This study may inspire insights into novel therapeutic strategies of NAFLD and provide references for future researches. Nevertheless, further experiments are still required to validate the findings of this study.

\section{Data Availability}

All the data used to support the findings of this study are included in the article or could be accessed in the open online databases mentioned in the Methods section.

\section{Conflicts of Interest}

The authors declare that there are no conflicts of interest.

\section{Supplementary Materials}

Supplementary Table 1: information of all the bioactive compounds of DNt. Supplementary Table 2: the potential targets of DNt in the treatment of NAFLD (Supplementary Materials)

\section{References}

[1] Z. M. Younossi, A. B. Koenig, D. Abdelatif, Y. Fazel, L. Henry, and M. Wymer, "Global epidemiology of nonalcoholic fatty liver disease-Meta-analytic assessment of prevalence, incidence, and outcomes," Hepatology, vol. 64, no. 1, pp. 73-84, 2016.

[2] A. M. Diehl and C. Day, "Cause, pathogenesis, and treatment of nonalcoholic steatohepatitis," New England Journal of Medicine, vol. 377, no. 21, pp. 2063-2072, 2017.

[3] S. Schuster, D. Cabrera, M. Arrese, and A. E. Feldstein, "Triggering and resolution of inflammation in NASH," $\mathrm{Na}$ ture Reviews Gastroenterology \& Hepatology, vol. 15, no. 6, pp. 349-364, 2018.

[4] Y. Rvinen, "Non-alcoholic fatty liver disease as a cause and a consequence of metabolic syndrome," Lancet Diabetes \& Endocrinology, vol. 2, no. 11, pp. 901-910, 2014.
[5] C. D. Byrne and G. Targher, "NAFLD: a multisystem disease," Journal of Hepatology, vol. 62, no. 1, pp. S47-S64, 2015.

[6] M. E. Rinella, "Nonalcoholic fatty liver disease," JAMA, vol. 313, no. 22, pp. 2263-2273, 2015.

[7] G. Musso, R. Gambino, M. Cassader, and G. Pagano, "A metaanalysis of randomized trials for the treatment of nonalcoholic fatty liver disease," Hepatology, vol. 52, no. 1, pp. 79-104, 2010.

[8] Y. Takahashi, K. Sugimoto, H. Inui et al., "Current pharmacological therapies for nonalcoholic fatty liver disease/ nonalcoholic steatohepatitis," World Journal of Gastroenterology, vol. 21, no. 13, pp. 3777-3785, 2015.

[9] J. G. Fan, "Evaluating the efficacy and safety of Danning Pian in the short-term treatment of patients with non-alcoholic fatty liver disease: a multicenter clinical trial," Hepatobiliary \& Pancreatic Diseases International: HBPD INT, vol. 3, no. 3, pp. 375-380, 2004.

[10] Y. Wang and H. Qi, "Clinical study on Danning Tablets combined with tolynicate and naphthylacetic acid in treatment of non-alcoholic fatty liver," Drugse Clinic (In Chinese), vol. 34, no. 1, pp. 88-92, 2019.

[11] W. Du, Q. Ye, J. Li et al., "Changes of blood liver function index and TNF- $\alpha$ levels before and after treatment of patients with nonalcoholic fatty liver disease with combination therapy of DanNing tablets and simvastatin," Journal of Practical Hepatology (In Chinese), vol. 20, no. 4, pp. 490-491, 2017.

[12] J. Zhang, X. Wang, N. Jiang et al., "Therapeutic eff ect of Danning tablets on nonalcoholic fatty liver disease in rats," World Chinese Journal of Digestology (In Chinese), vol. 24, no. 18, pp. 2875-2880, 2016.

[13] R. Liu, Z. Chen, R. Xu et al., "The protective effect of Danning tablet on experimental fatty liver," Pharmaceutical Care and Research, vol. 7, no. 3, pp. 202-205, 2007, in Chinese.

[14] Y. Yang, P. Zhu, J. Zhang, X. Zhang, and B. Fang, "Effects of Danning tablets on expression of PPAR $\alpha$ and CYP7A1 in rats with nonalcoholic fatty liver disease," Chinese Journal of New Drugs and Clinical Remedies (In Chinese), vol. 26, no. 10, pp. 721-726, 2007.

[15] L. Ding, B. Zhang, C. Zhan et al., "Danning tablets attenuates alpha-naphthylisothiocyanate- induced cholestasis by modulating the expression of transporters and metabolic enzymes," BMC Complementary and Alternative Medicine, vol. 14, no. 17, p. 249, 2014.

[16] L. Lu, F. Zhang, C. Zhan et al., "The protective effect of Danning tablet against hepatic sinusoidal obstruction syndrome induced by Gynura Rhizoma," Acta Pharmaceutica Sinica (In Chinese), vol. 54, no. 3, pp. 494-501, 2019.

[17] L.-L. Ding, B.-F. Zhang, W. Dou, L. Yang, C.-S. Zhan, and Z.-T. Wang, "Protective effect of Danning tablet on acute livery injury with cholestasis induced by $\alpha$-naphthylisothiocyanate in rats," Journal of Ethnopharmacology, vol. 140, no. 2, pp. 222-229, 2012.

[18] L. Pinzi and G. Rastelli, "Molecular docking: shifting paradigms in drug discovery," International Journal of Molecular Sciences, vol. 20 , no. 18, p. 4331, 2019.

[19] J. Ru, P. Li, J. Wang et al., "TCMSP: a database of systems pharmacology for drug discovery from herbal medicines," Journal of Cheminformatics, vol. 6, no. 16, p. 13, 2014.

[20] L. Huang, D. Xie, Y. Yu et al., “Tcmid 2.0: a comprehensive resource for TCM," Nucleic Acids Research, vol. 46, no. D1, pp. D1117-D1120, 2018.

[21] X. Xu, W. Zhang, C. Huang et al., "A novel chemometric method for the prediction of human oral bioavailability," 
International Journal of Molecular Sciences, vol. 13, no. 6, pp. 6964-6982, 2012.

[22] T. J. Flynn and S. N. Vohra, "Simultaneous determination of intestinal permeability and potential drug interactions of complex mixtures using Caco-2 cells and high-resolution mass spectrometry: studies with Rauwolfia serpentina extract," Chemico-Biological Interactions, vol. 290, no. 25, pp. 37-43, 2018.

[23] A. I. Casas, A. A. Hassan, S. J. Larsen et al., "From single drug targets to synergistic network pharmacology in ischemic stroke," Proceedings of the National Academy of Sciences, vol. 116, no. 14, pp. 7129-7136, 2019.

[24] W. Tao, X. Xu, X. Wang et al., "Network pharmacology-based prediction of the active ingredients and potential targets of Chinese herbal Radix Curcumae formula for application to cardiovascular disease," Journal of Ethnopharmacology, vol. 145, no. 1, pp. 1-10, 2013.

[25] S. Kim, J. Chen, T. Cheng et al., "PubChem 2019 update: improved access to chemical data," Nucleic Acids Research, vol. 47, no. D1, pp. D1102-D1109, 2019.

[26] A. Daina, O. Michielin, and V. Zoete, "SwissTargetPrediction: updated data and new features for efficient prediction of protein targets of small molecules," Nucleic Acids Research, vol. 47, no. W1, pp. W357-W364, 2019.

[27] X. Wang, Y. Shen, S. Wang et al., "PharmMapper 2017 update: a web server for potential drug target identification with a comprehensive target pharmacophore database," Nucleic Acids Research, vol. 45, no. W1, pp. W356-W360, 2017.

[28] N. Rappaport, S. Fishilevich, R. Nudel et al., "Rational confederation of genes and diseases: NGS interpretation via GeneCards, MalaCards and VarElect," Biomedical Engineering Online, vol. 16, no. S1, p. 72, 2017.

[29] J. Pinero, J. M. Ramirez-Anguita, J. Sauch-Pitarch et al., "The DisGeNET knowledge platform for disease genomics: 2019 update," Nucleic Acids Research, vol. 48, no. D1, pp. D845D855, 2019.

[30] A. P. Davis, C. J. Grondin, R. J. Johnson et al., "The comparative Toxicogenomics database: update 2019," Nucleic Acids Research, vol. 47, no. D1, pp. D948-D954, 2019.

[31] A. Hamosh, A. F. Scott, J. S. Amberger, C. A Bocchini, and V. A McKusick, "Online Mendelian Inheritance in Man (OMIM), a knowledgebase of human genes and genetic disorders," Nucleic Acids Research, vol. 33, no. 1, pp. D514-D517, 2005.

[32] D. Szklarczyk, J. H. Morris, H. Cook et al., "The STRING database in 2017: quality-controlled protein-protein association networks, made broadly accessible," Nucleic Acids Research, vol. 45, no. D1, pp. D362-D368, 2017.

[33] P. Shannon, A. Markiel, O. Ozier et al., "Cytoscape: a software environment for integrated models of biomolecular interaction networks," Genome Research, vol. 13, no. 11, pp. 2498-2504, 2003.

[34] J.-M. Yang and C.-C. Chen, "GEMDOCK: a generic evolutionary method for molecular docking," Proteins: Structure, Function, and Bioinformatics, vol. 55, no. 2, pp. 288-304, 2004.

[35] H. M. Berman, J. D. Westbrook, Z. Feng, G. Gilliland, and P. E. Bourne, "The protein Data Bank," Nucleic Acids Research, vol. 28, no. 1, pp. 235-242, 2000.

[36] T. Sterling and J. J. Irwin, "Zinc 15 - ligand discovery for everyone," Journal of Chemical Information and Modeling, vol. 55, no. 11, pp. 2324-2337, 2015.

[37] S. Pisonero-Vaquero, Á. Martínez-Ferreras, M. V. GarcíaMediavilla et al., "Quercetin ameliorates dysregulation of lipid metabolism genes via the PI3K/AKT pathway in a diet-induced mouse model of nonalcoholic fatty liver disease," Molecular Nutrition \& Food Research, vol. 59, no. 5, pp. 879-893, 2015.

[38] M. Tamura, H. Nakagawa, S. Hori, T. Suzuki, and K. Hirayama, "Plasma quercetin metabolites are affected by intestinal microbiota of human microbiota-associated mice fed with a quercetin-containing diet," Journal of Clinical Biochemistry and Nutrition, vol. 65, no. 3, pp. 232-239, 2019.

[39] D. Porras, E. Nistal, S. Martínez-Flórez et al., "Protective effect of quercetin on high-fat diet-induced non-alcoholic fatty liver disease in mice is mediated by modulating intestinal microbiota imbalance and related gut-liver axis activation," Free Radical Biology and Medicine, vol. 102, pp. 188-202, 2017.

[40] E.-Y. Kwon, U. J. Jung, T. Park, J. W. Yun, and M.-S. Choi, "Luteolin attenuates hepatic steatosis and insulin resistance through the interplay between the liver and adipose tissue in mice with diet-induced obesity," Diabetes, vol. 64, no. 5, pp. 1658-1669, 2015.

[41] E. Y. Kwon and M. S. Choi, "Luteolin targets the toll-like receptor signaling pathway in prevention of hepatic and adipocyte fibrosis and insulin resistance in diet-induced obese mice," Nutrients, vol. 10, no. 10, p. 1415, 2018.

[42] M.-H. Hoang, Y. Jia, B. Mok, H.-j. Jun, K.-Y. Hwang, and S.-J. Lee, "Kaempferol ameliorates symptoms of metabolic syndrome by regulating activities of liver $\mathrm{X}$ receptor- $\beta$," The Journal of Nutritional Biochemistry, vol. 26, no. 8, pp. 868$875,2015$.

[43] V. Francisco, A. Figueirinha, G. Costa et al., "The flavone luteolin inhibits liver X receptor activation," Journal of Natural Products, vol. 79, no. 5, pp. 1423-1428, 2016.

[44] Y. Yin, L. Gao, H. Lin et al., "Luteolin improves non-alcoholic fatty liver disease in $\mathrm{db} / \mathrm{db}$ mice by inhibition of liver $\mathrm{X}$ receptor activation to down-regulate expression of sterol regulatory element binding protein 1c," Biochemical and Biophysical Research Communications, vol. 482, no. 4, pp. 720-726, 2017.

[45] H. T. M. Hien, N. C. Ha, L. T. Thom, and D. D. Hong, "Squalene promotes cholesterol homeostasis in macrophage and hepatocyte cells via activation of liver X receptor (LXR) $\alpha$ and $\beta$," Biotechnology Letters, vol. 39, no. 8, pp. 1101-1107, 2017.

[46] S. Feng, L. Gan, C. S. Yang et al., "Effects of stigmasterol and $\beta$-sitosterol on nonalcoholic fatty liver disease in a mouse model: a lipidomic analysis," Journal of Agricultural and Food Chemistry, vol. 66, no. 13, pp. 3417-3425, 2018.

[47] S. Feng, Z. Dai, A. B. Liu et al., "Intake of stigmasterol and $\beta$-sitosterol alters lipid metabolism and alleviates NAFLD in mice fed a high-fat western-style diet," Biochimica et Biophysica Acta (BBA) - Molecular and Cell Biology of Lipids, vol. 1863, no. 10, pp. 1274-1284, 2018.

[48] H. Matsuda, T. Morikawa, K. Ninomiya, and M. Yoshikawa, "Hepatoprotective constituents from zedoariae rhizoma: absolute stereostructures of three new carabrane-type sesquiterpenes, curcumenolactones A, B, and C," Bioorganic \& Medicinal Chemistry, vol. 9, no. 4, pp. 909-916, 2001.

[49] K.-Q. Shi, Y.-C. Fan, W.-Y. Liu, L.-F. Li, Y.-P. Chen, and M.-H. Zheng, "Traditional Chinese medicines benefit to nonalcoholic fatty liver disease: a systematic review and metaanalysis," Molecular Biology Reports, vol. 39, no. 10, pp. 9715-9722, 2012.

[50] T.-p. Li, R.-g. Zhu, Y.-p. Dong, Y.-h. Liu, S.-h. Li, and G. Chen, "Effects of pectin pentaoligosaccharide from hawthorn (crataegus pinnatifida bunge. Var. Major) on the activity and mRNA levels of 
enzymes involved in fatty acid oxidation in the liver of mice fed a high-fat diet," Journal of Agricultural and Food Chemistry, vol. 61, no. 31, pp. 7599-7605, 2013.

[51] W.-H. Hu, G. K.-L. Chan, J.-S. Lou et al., "The extract of Polygoni Cuspidati Rhizoma et Radix suppresses the vascular endothelial growth factor-induced angiogenesis," Phytomedicine, vol. 42, pp. 135-143, 2018.

[52] J. Jiang, L. Yan, Z. Shi, L. Wang, L. Shan, and T. Efferth, "Hepatoprotective and anti-inflammatory effects of total flavonoids of Qu Zhi Ke (peel of Citrus changshan-huyou) on non-alcoholic fatty liver disease in rats via modulation of NF$\kappa \mathrm{B}$ and MAPKs," Phytomedicine, vol. 64, no. 11, p. 153082, 2019.

[53] F. Yang, Y. Xu, A. Xiong et al., "Evaluation of the protective effect of Rhei Radix et Rhizoma against $\alpha$-naphthylisothiocyanate induced liver injury based on metabolic profile of bile acids," Journal of Ethnopharmacology, vol. 144, no. 3, pp. 599-604, 2012.

[54] X. R. Zhou, J. H. Wang, B. Jiang, J. Shang, and C. Q. Zhao, “A study of extraction process and in vitro antioxidant activity of total phenols from Rhizoma Imperatae," African Journal of Traditional, Complementary, and Alternative Medicines, vol. 10, no. 4, pp. 175-178, 2013.

[55] I. Park, J. Ryuk, H. Lee, H. Go, and B. Ko, "In vitro and in vivo effects of ethanol extract combined with Curcumae Radix and Glycyrrhizae Radix et Rhizoma on menopausal metabolic disturbances," International Journal of Clinical and Experimental Medicine, vol. 8, no. 9, pp. 15076-15086, 2015.

[56] K. Asadipooya, K. B. Lankarani, R. Raj et al., "RAGE is a potential cause of onset and progression of nonalcoholic fatty liver disease," International Journal of Endocrinology, vol. 2019, Article ID 2151302, 11 pages, 2019.

[57] S. Miltonprabu, M. Tomczyk, K. Skalicka-Woźniak et al., "Hepatoprotective effect of quercetin: from chemistry to medicine," Food and Chemical Toxicology, vol. 108, no. Pt B, pp. 365-374, 2017.

[58] T. Ezure, T. Sakamoto, H. Tsuji et al., "The development and compensation of biliary cirrhosis in interleukin-6-deficient mice," The American Journal of Pathology, vol. 156, no. 5, pp. 1627-1639, 2000.

[59] D. M. S. Ferreira, R. E. Castro, M. V. Machado et al., "Apoptosis and insulin resistance in liver and peripheral tissues of morbidly obese patients is associated with different stages of non-alcoholic fatty liver disease," Diabetologia, vol. 54, no. 7, pp. 1788-1798, 2011.

[60] S. Coulon, S. Francque, I. Colle et al., "Evaluation of inflammatory and angiogenic factors in patients with non-alcoholic fatty liver disease," Cytokine, vol. 59, no. 2, pp. 442-449, 2012.

[61] A. Lonardo, F. Nascimbeni, A. Mantovani, and G. Targher, "Hypertension, diabetes, atherosclerosis and NASH: cause or consequence?" Journal of Hepatology, vol. 68, no. 2, pp. 335-352, 2018.

[62] K. Wang, "Molecular mechanisms of hepatic apoptosis," Cell Death \& Disease, vol. 5, no. 1, p. e996, 2014.

[63] Z. Chen, R. Yu, Y. Xiong et al., "A vicious circle between insulin resistance and inflammation in nonalcoholic fatty liver disease," Lipids in Health and Disease, vol. 16, no. 1, 2017.

[64] T. Liu, Y. Zhou, K. S. Ko et al., "Interactions between Myc and mediators of inflammation in chronic liver diseases," Mediators of Inflammation, vol. 2015, Article ID 276850, 2015.

[65] G. Verdile, K. N. Keane, V. F. Cruzat et al., "Inflammation and oxidative stress: the molecular connectivity between insulin resistance, obesity, and Alzheimer's disease," Mediators of Inflammation, vol. 2015, Article ID 105828, 17 pages, 2015.
[66] Y. Zhang, B. Zhou, B. Deng et al., “Amyloid- induces hepatic insulin resistance in vivo via JAK2," Diabetes, vol. 62, no. 4, pp. 1159-1166, 2013.

[67] V. Arroyo, R. García-Martinez, and X. Salvatella, "Human serum albumin, systemic inflammation, and cirrhosis," Journal of Hepatology, vol. 61, no. 2, pp. 396-407, 2014.

[68] C. Bocca, E. Novo, A. Miglietta, and M. Parola, "Angiogenesis and fibrogenesis in chronic liver diseases," Cellular and Molecular Gastroenterology and Hepatology, vol. 1, no. 5, pp. 477-488, 2015.

[69] J. Skat-Rørdam, D. Højland Ipsen, and J. Lykkesfeldt, “A role of peroxisome proliferator-activated receptor $\gamma$ in non-alcoholic fatty liver disease," Basic \& Clinical Pharmacology \& Toxicology, vol. 124, no. 5, pp. 528-537, 2019.

[70] C. J. Villanueva and P. Tontonoz, "Licensing PPAR $\gamma$ to work in macrophages," Immunity, vol. 33, no. 5, pp. 647-649, 2010.

[71] Z. Wang, J.-P. Xu, Y.-C. Zheng et al., "Peroxisome proliferator-activated receptor gamma inhibits hepatic fibrosis in rats," Hepatobiliary \& Pancreatic Diseases International, vol. 10, no. 1, pp. 64-71, 2011. 